\title{
A Novel Animal Model of Parkinson's Disease Using Optogenetics: Representation of Various Disease Stages by Modulating the Illumination Parameter
}

\author{
Eun Jung Lee ${ }^{a}$ Hyung Ho Yoon ${ }^{b}$ Eun Suk Park ${ }^{c}$ Joongkee Min ${ }^{b}$ \\ Sang Ryong Jeon ${ }^{b}$ \\ a Department of Neurosurgery, Hallym University Dongtan Sacred Heart Hospital, Hwaseong-si, Republic of \\ Korea; ${ }^{b}$ Department of Neurological Surgery, Asan Medical Center, University of Ulsan College of Medicine,

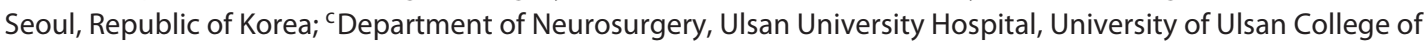 \\ Medicine, Ulsan, Republic of Korea
}

\section{Keywords}

Animal model · Halorhodopsin · Optogenetics · Parkinson's disease

\begin{abstract}
Background: The classic animal model of Parkinson's disease (PD) using neurotoxin can only simulate fixed stages of the disease by causing irreversible damage to the nigrostriatal system. Objectives: To develop an optogenetic PD model that can modulate the severity of disease by optical stimulation by introducing the halorhodopsin (NpHR) gene into the substantia nigra compacta. Methods: Fifteen rats received injections of engineered AAV with NpHR-YFP gene into the substantia nigra. They were then subjected to illumination of 590-nm light wavelengths with 3 optical stimulation conditions, i.e., frequency-width: $5 \mathrm{~Hz}-10 \mathrm{~ms}(n=5)$, $5 \mathrm{~Hz}-100 \mathrm{~ms}(n=5)$, and $50 \mathrm{~Hz}-10 \mathrm{~ms}(n=5)$. Eleven rats received 6-hydroxydopamine injections to establish the conventional PD model. Results: The optogenetic models showed characteristic PD manifestations, similar to those of the conventional models; the severity of forelimb akinesia
\end{abstract}

correlated with the total illumination value (frequency $x$ width). The group with a low illumination value ( $5 \mathrm{~Hz}-10 \mathrm{~ms}$ ) was comparable to the conventional partial model whereas the groups with high illumination values $(5 \mathrm{~Hz}-100 \mathrm{~ms}$ and $50 \mathrm{~Hz}-10 \mathrm{~ms}$ ) were similar to the conventional complete model. Conclusions: An optogenetic PD model has the advantage of more appropriately representing various PD stages by controlling illumination parameters.

๑) 2018 S. Karger AG, Basel

\section{Introduction}

Parkinson's disease (PD) is a common and slowly progressive neurodegenerative disease caused by the gradual loss of dopaminergic (DAergic) neurons in the substantia nigra pars compacta (SNc). Resting tremor, bradykinesia, and rigidity are cardinal presenting symptoms. The abnormal electrical activity in the cortico-striatopallido-

Eun Jung Lee and Hyung Ho Yoon contributed equally to this work.

\section{KARGER}

(c) 2018 S. Karger AG, Basel

E-Mail karger@karger.com

www.karger.com/sfn
Sang Ryong Jeon, $\mathrm{MD}, \mathrm{PhD}$

Department of Neurological Surgery, Asan Medical Center

University of Ulsan College of Medicine

88 Olympic-ro 43-gil, Songpa-gu, Seoul 05505 (Republic of Korea)

E-Mail srjeon@amc.seoul.kr 
thalamo-cortical motor circuit caused by the loss of DAergic neurons is a known pathophysiology of PD [13]; however, the most disrupted brain segment in this circuit remains to be completely clarified. Animal experiments are suitable to further clarify the pathogenesis and progression of $\mathrm{PD}$ as well as to study appropriate and new therapeutic targets and approaches. Animal models should reflect the state of the disease to be studied as closely as possible.

One of the classic PD models in rats was made by injecting 6-hydroxydopamine (6-OHDA), a common neurotoxin that induces the selective degeneration of catecholaminergic neurons in the substantia nigra (SN) and medial forebrain bundle (MFB) or striatum [4-6]. When 6-OHDA is injected into the MFB or $\mathrm{SN}$, rapid and near-complete DAergic cell death in the SN occurs within 1 week, representing advanced-stage PD (the complete PD model) [4, 7]. On the other hand, an injection of 6-OHDA into the striatum partially destroys the axons and terminals of DAergic neurons within a circumscribed area surrounding the injection site in a slow and delayed manner over 4-8 weeks (the partial PD model), and causes motor symptoms corresponding to early-to-middle-stage PD [8-11]. However, using conventional 6-OHDA injections precludes the establishment of a model that represents the various stages of PD. The partial model, in particular, has the added disadvantages of taking a long time to create, and there are challenges associated with the locational accuracy of lesions required to elicit the desired PD symptoms $[8,12]$. Meanwhile, there are several genetic models using transgenic mice and rats that are manipulated to bear mutations in specific PD-related genes. To date, more than 20 genes have already been linked to an elevated risk of PD, and these genetic models have proven useful in exploring the functions and clinical implications of particular genes [1317]. However, it is difficult to create a model that encompasses such diverse genetic aspects of the disease, and a genetic model does not represent PD in general because only $10-20 \%$ of PD is due to genetic causes $[14,18,19]$.

A recent development in optogenetics, which introduces the gene of a light-activated ion channel into specific cells infected with the engineered virus, has enabled the activation or inhibition of neurons via the depolarization or hyperpolarization of membrane potential, respectively, under a certain wavelength of light [20,21]. It has been reported that cellular activity controls various intracellular signaling pathways [22-24] by recruiting signaling proteins to or away from a specific intracellular site of action, resulting in signal activation or inhibition $[25,26]$. Furthermore, it has been reported that cellular activity can be adjusted to various levels by fine-tuning parameters of illumination such as frequency and width [27]. We previously studied the possibility of optogenetics as an alternative to deep brain stimulation in the treatment of PD by expressing the inhibitory optogene, a halorhodop$\sin (N p H R)$ that transports a chloride ion into neurons and silences neuronal activity when activated with yellow light (i.e., yellow fluorescent protein [YFP]) $[28,29]$. $N p H R$ was introduced into the subthalamic nucleus of a 6-OHDA-induced rat model of $\mathrm{PD}$, and we demonstrated that decreasing the hyperactivity of the subthalamic nucleus with yellow-light illumination improves forelimb akinesia and L-DOPA-induced dyskinesia.

Based on these previous findings, we thought of developing a PD model that could simulate the various disease stages by introducing the NpHR gene into DAergic neurons in the $\mathrm{SN}$, and then adjusting the illumination parameters. We aimed to: (1) investigate whether a model using NpHR injections could induce appropriate motor symptoms of PD and the severity of motor symptoms could be modulated by optical-inhibition conditions, (2) compare the disease severity of the optogenetic models under different optical conditions with the conventional PD model using 6-OHDA injections, and (3) clarify the mechanism of PD manifestation in optogenetic models by analyzing immunofluorescence staining patterns.

\section{Materials and Methods}

\section{Experimental Animals}

A total of 31 male Wistar rats (Orient Bio Inc., Seongnam, Republic of Korea), weighing 300-350 g at the beginning of the experiment, were housed in a room with a 12-h light/dark cycle and free access to food and water. All procedures complied with the guidelines of the Institutional Animal Care and Use Committee of the Asan Institute for Life Sciences (Seoul, Republic of Korea).

\section{Experimental Procedures}

The timelines of the experiments are presented in Figure 1. In experiment 1, 20 rats received engineered viral injections. Fifteen rats received hSynapsin1-NpHR-YFP adeno-associated virus (AAV) injections targeted to the right $\mathrm{SN}$. They were separated into 3 groups with a frequency-width of $5 \mathrm{~Hz}-10 \mathrm{~ms}(n=5), 5 \mathrm{~Hz}$ $100 \mathrm{~ms}(n=5)$, and $50 \mathrm{~Hz}-10 \mathrm{~ms}(n=5)$. The remaining 5 rats received hSynapsin1-YFP AAV injections into the right $\mathrm{SN}$ as a control group. All rats were subjected to optical fiber insertions, illumination with 590-nm light, and stepping tests. In experiment 2, 5 rats received 6-OHDA injections into the right MFB. After 1-2 weeks, they were subjected to apomorphine rotation tests to screen for complete lesions, and were then subjected to stepping tests. In experiment 3, 6 rats received 6-OHDA injections into the right striatum to establish partial lesions of $\mathrm{PD}$, and they were then subjected to serial stepping tests. 
Fig. 1. Experimental procedures. Experiment 1: 20 rats each received virus injections (hSynapsin1-NpHR-YFP AAV or hSynapsin1-YFP AAV) that were targeted to the right substantia nigra. They were separated into 4 groups: $5 \mathrm{~Hz}-10 \mathrm{~ms}(n=$ 5), $5 \mathrm{~Hz}-100 \mathrm{~ms}(n=5), 50 \mathrm{~Hz}-10 \mathrm{~ms}(n=$ $5)$, and a control group $(n=5)$. All rats were subjected to optical fiber insertion, illumination with 590-nm light, and stepping tests. Experiment 2: 5 rats received 6-OHDA injections into the right medial forebrain bundle and were subjected to stepping tests. Experiment 3: 6 rats received 6-OHDA injections into the right striatum to generate a partial lesion of Parkinson disease and were then subjected to stepping tests. Pre, prior to illumination; Light, $16 \mathrm{~h}$ after illumination under the light-on condition; Post, ( 2 or 4 weeks [ $2 \mathrm{w}$ or $4 \mathrm{w}$ ]) after illumination, i.e., under the light-off condition.

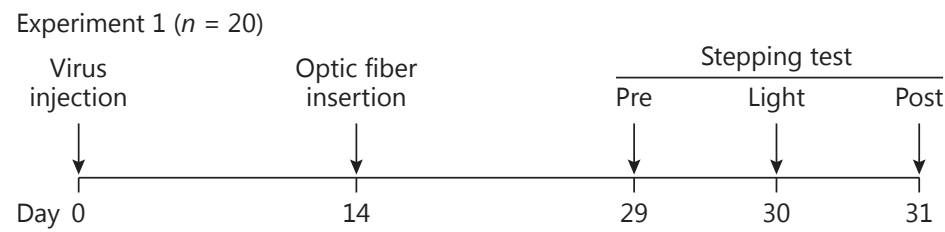

Experiment $2(n=5)$

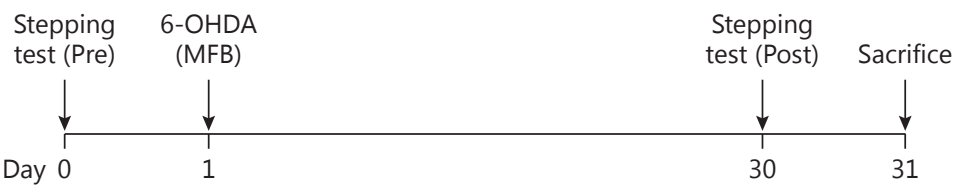

Experiment $3(n=6)$

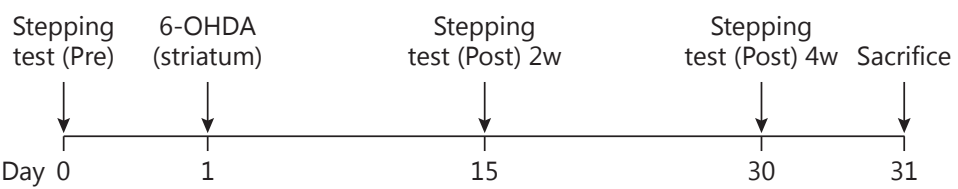

Preparation of hSynapsin1-NpHR-YFP AAV and hSynapsin1YFP AAV

Viruses were prepared as described previously [29]. Briefly, the AAV plasmid vector pAAV2-hSynapsin1-NpHR-YFP was constructed by removing the CMV promoter from pAAV2-CMV (Stratagene, La Jolla, CA, USA) and inserting the hSynapsin 1 promoter with the NpHR-YFP sequence (plasmid \#26775, Addgene). pAAV2-hSynapsin1-YFP AAV was used as a control vector with only the YFP sequence instead of the NpHR-YFP sequence. The pAAV-RC plasmid harbors the AAV rep and cap genes, encoding the replication and virus capsid structure proteins, respectively. The pHelper plasmid contains the essential subset of adenovirus genes, $V A, E 2 A$, and $E 4$, necessary for AAV production in HEK293 cells. For AAV2/2 production, 3 AAV plasmids ( $10 \mu \mathrm{g}$ of each plasmid) were cotransfected into HEK293 cells using a polyethylenimine transfection kit (Polyplus, Illkirch-Graffenstaden, France).

\section{Stereotactic AAV Injection}

The number of viral particles was a minimum of $1.0 \times 10^{10} / \mathrm{mL}$, which is considered a concentrated virus package. Viral injections were performed under general anesthesia with an intraperitoneal injection of a mixture of $35 \mathrm{mg} / \mathrm{kg}$ zolazepam and tiletamine (Zoletil, Virbac S.A., Carros, France) and $5 \mathrm{mg} / \mathrm{kg}$ xylazine (Rompun, Bayer, Leverkusen, Germany). Twenty rats each received $2.0-\mu \mathrm{L}$ injections of hSynapsin1-NpHR-YFP AAV $(n=15)$ or hSynapsin1-YFP AAV $(n=5)$ that targeted the right SN (coordinates: anterior-posterior $[\mathrm{AP}]-5.6 \mathrm{~mm}$, lateral $[\mathrm{L}]+2.0 \mathrm{~mm}$ relative to bregma, and ventral $[\mathrm{V}]-7.5 \mathrm{~mm}$ from the dura). The AAV was delivered at a rate of $0.2 \mu \mathrm{L} / \mathrm{min}$ using a Hamilton syringe and an automated microsyringe pump (Harvard Apparatus, Holliston, MA, USA). After injection, the needle was kept in place for $5 \mathrm{~min}$ to prevent the solution from flowing backward, and was then retracted over the next $5 \mathrm{~min}$.

Modulating the Illumination Parameter in an Animal Model to Represent PD Stages

\section{Optical Fiber Insertion and Light Stimulation}

Optical fibers (core $200 \mu \mathrm{m}$, outer diameter $245 \mu \mathrm{m}$, numerical aperture 0.53, RM3 type, flat tip; Doric Lenses, Québec, QC, Canada) were optimized to the depth of the SN by cutting into $8.3-\mathrm{mm}$ lengths. Two weeks after the AAV injections, each rat was positioned in a stereotactic frame under general anesthesia. After a scalp incision, 4 burr holes were drilled to embed screws around the insertion site of the optical fiber. The optical fiber was implanted using a stereotactic cannula holder into the SN, and then firmly fixed with surrounding screws using dental cement (Vertex, Zeist, The Netherlands). For the illumination, the implanted optical fiber was connected to a fiber-optic rotary joint and an LED fiber-optic light source (Doric lenses) that produced amber (590$\mathrm{nm}$ ) light. According to the manufacturer's datasheet, output power with a $200-\mu \mathrm{m}$ fiber core is $1.2 \mathrm{~mW}$ and spectral bandwidth 16 $\mathrm{nm}$ FWHM when amber light $(590 \mathrm{~nm})$ is illuminated. Each combination parameter $(5 \mathrm{~Hz}-10 \mathrm{~ms}, 5 \mathrm{~Hz}-100 \mathrm{~ms}$, and $50 \mathrm{~Hz}-10 \mathrm{~ms}$ ) was applied under the control of a pulse generator (Berkeley $\mathrm{Nu}$ cleonics Corp., San Rafael, CA, USA) with a transistor-transistor logic signal. Optic inhibition was executed in freely moving animals in glass cylinders (diameter $20 \mathrm{~cm}$, height $40 \mathrm{~cm}$ ) for a total of $16 \mathrm{~h}$ over 2 days. Specifically, the rats came under illumination for $8 \mathrm{~h}$ per each day with a 12 -h rest period after the 8 -h stimulation on the first day.

\section{6-OHDA Lesion Surgery}

Surgical procedures were performed under general anesthesia induced by an intraperitoneal injection of a mixture of $35 \mathrm{mg} / \mathrm{kg}$ zolazepam and tiletamine and $5 \mathrm{mg} / \mathrm{kg}$ xylazine. Five rats received unilateral injections of $8 \mu \mathrm{g}$ of 6-OHDA (Sigma, St. Louis, MO, USA) in $4 \mu \mathrm{L}$ of $0.9 \%$ saline with $0.1 \%$ ascorbic acid into the right MFB at the following coordinates: AP $-2.2 \mathrm{~mm}, \mathrm{~L}+1.5 \mathrm{~mm}$ relative to the bregma, and $\mathrm{V}-8.0 \mathrm{~mm}$ from the dura, with the tooth bar set at $+4.5 \mathrm{~mm}$ [30]. Six rats received unilateral injections of $7 \mu \mathrm{g}$ of 


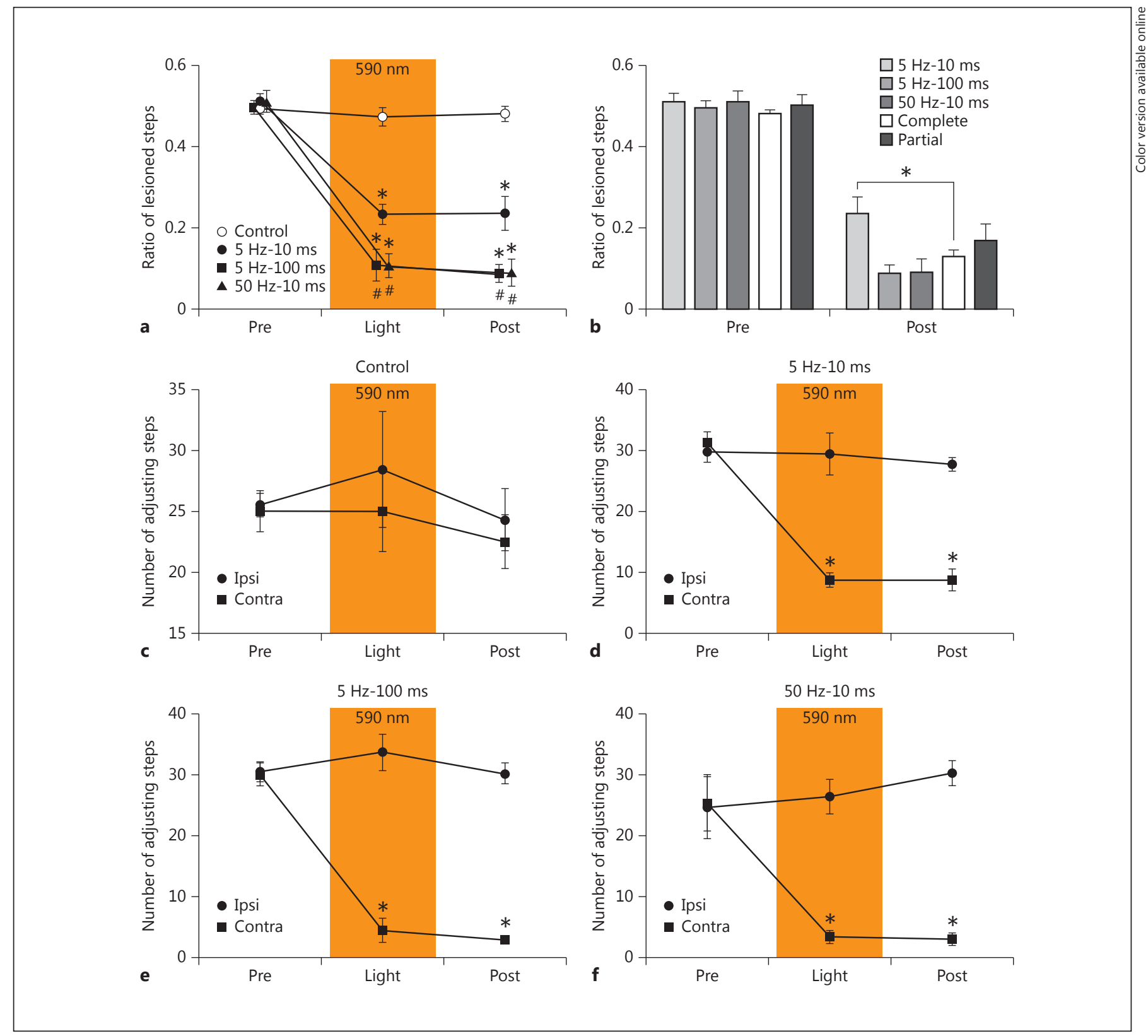

Fig. 2. Stepping tests. a The $5 \mathrm{~Hz}-10 \mathrm{~ms}, 5 \mathrm{~Hz}-100 \mathrm{~ms}$, and $50 \mathrm{~Hz}-$ $10 \mathrm{~ms}$ groups showed significant decreases in the ratio of the lesioned forelimb steps compared to the control groups after $16 \mathrm{~h}$ of illumination with 590-nm light (Light) or $24 \mathrm{~h}$ after lights were switched off (Post) $\left.*^{*} p<0.001\right)$. The groups with a higher frequency $(50 \mathrm{~Hz}-10 \mathrm{~ms})$ or greater width $(5 \mathrm{~Hz}-100 \mathrm{~ms})$ showed more severe akinesia under the Light $\left({ }^{\#} p<0.05\right)$ and Post $\left({ }^{\#} p<\right.$ 0.01 ) conditions. b The $5 \mathrm{~Hz}-10 \mathrm{~ms}$ group did not show a significant difference from the 6-OHDA-induced partial model group, but was significantly different from the 6-OHDA-induced com- plete model group $(* p<0.05)$. By contrast, the $5 \mathrm{~Hz}-100 \mathrm{~ms}$ and $50 \mathrm{~Hz}-10 \mathrm{~ms}$ groups showed no significant difference in comparison to both the 6-OHDA partial model and complete model groups. d-f The $5 \mathrm{~Hz}-10 \mathrm{~ms}, 5 \mathrm{~Hz}-100 \mathrm{~ms}$, and $50 \mathrm{~Hz}-10 \mathrm{~ms}$ groups showed significant decreases in contralateral (Contra) steps compared to ipsilateral (Ipsi) steps $\left({ }^{*} p<0.001\right)$. c However, the control group did not show contralateral forelimb akinesia after $16 \mathrm{~h}$ of illumination with 590-nm light. c-f These persisted a day after the light was switched off. Two-way repeated-measures ANOVA with the Bonferroni post hoc test. Pre, prior to illumination. 
6-OHDA in $3 \mu \mathrm{L}$ of $0.9 \%$ saline with $0.1 \%$ ascorbic acid into the right striatum at the following coordinates: $\mathrm{AP}+1.2 /+0.2 \mathrm{~mm}, \mathrm{~L}+2.5 / 3.0$ $\mathrm{mm}$, and $\mathrm{V}-5.0 \mathrm{~mm}$ from the dura [31]. The toxin was delivered at a rate of $0.7 \mu \mathrm{L} / \mathrm{min}$ using a 33-gauge Hamilton syringe and an automated microsyringe pump (Harvard Apparatus). After injection, the needle was kept in place for $5 \mathrm{~min}$ to prevent the solution from flowing backward, and was then retracted over the next $5 \mathrm{~min}$.

\section{Stepping Test}

In experiment 1, stepping tests were performed 3 times: prior to illumination (Pre), $16 \mathrm{~h}$ after illumination under the light-on condition (Light), and 1 day after illumination (Post), i.e., under the light-off condition (Fig. 1). In experiment 2, stepping tests were performed twice: Pre and 4 weeks Post. In experiment 3, stepping tests were performed twice: Pre and 4 weeks Post. The stepping test was performed as previously described with slight modifications $[29,32]$. Briefly, both hind limbs were held firmly in one hand of the experimenter and one forelimb in the other hand. The test was repeated with both the contralateral and ipsilateral forelimbs. The rostral part of the rat was lowered onto a treadmill (Jeung-Do Bio \& Plant Co., Seoul, Republic of Korea) that was moving at a rate of $1.8 \mathrm{~m} / 10 \mathrm{~s}$. The rat's body remained stationary while one forelimb was allowed to spontaneously touch the moving treadmill track for $10 \mathrm{~s}$. All experimental sessions were recorded by video to allow the number of adjusted steps taken to be counted. Every rat was subjected to the stepping test twice in each session, and the number of steps taken was averaged across the 2 trials.

\section{Tissue Processing}

For tissue fixation, rats were transcardially perfused with $0.9 \%$ saline containing 10,000 IU heparin (Hanlim Pharm, Seoul, Republic of Korea), followed by $4 \%$ paraformaldehyde in PBS. Brains were extracted and postfixed for $4 \mathrm{~h}$ in the same fixative, followed by dehydration in $30 \%$ sucrose until they sank. Coronal sections (40- $\mu \mathrm{m}$-thick) of the striatum (AP +2.04 to $-0.12 \mathrm{~mm}$ ) and SN (AP -4.8 to $-6.0 \mathrm{~mm}$ ) were collected using a cryotome (Thermo Scientific, Waltham, MA, USA) and then preserved under free-floating conditions in $0.08 \%$ sodium azide (Sigma) in PBS at $4{ }^{\circ} \mathrm{C}$.

\section{Immunohistochemical Staining}

Immunostaining procedures were performed as described below. Serial coronal sections of the SN or striatum were washed in 0.5\% BSA (Bioworld, Dublin, OH, USA) in PBS ( $\mathrm{pH} 7.4$ ), and then incubated with a blocking solution containing BSA, Triton X-100 (Sigma), and sodium azide (Sigma) in PBS. The sections were incubated overnight with a mouse anti-tyrosine hydroxylase $(\mathrm{TH})$ antibody (1:2,000; Sigma) in 0.5\% BSA in PBS (pH 7.4). They were subsequently incubated for $2 \mathrm{~h}$ with Alexa Fluor 555 donkey antimouse IgG (1:1,000; Invitrogen, Carlsbad, CA, USA). Fluorescentlabeled tissues were coverslipped with a fluorescent mounting medium (DAKO, Glostrup, Denmark).

\section{Imaging and Nigral Cell Counting}

Fluorescent images were obtained using a confocal microscope (Carl Zeiss, Oberkochen, Germany) with ZEN microscope software (Carl Zeiss). For nigral cell counting, TH-immunopositive cells on both sides of the SN were observed in 3 coronal sections. Data are expressed as the percentage of TH-positive cells in the ipsilateral SN compared with the number of TH-positive cells in the contralateral SN.

Modulating the Illumination Parameter in an Animal Model to Represent PD Stages

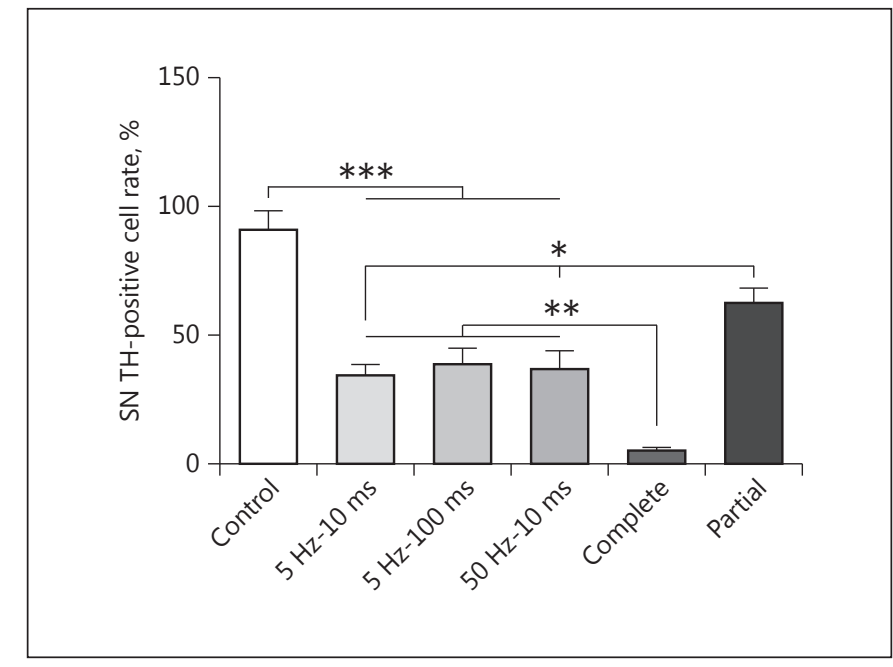

Fig. 3. Cell counts in the substantia nigra. The ipsilateral substantia nigra in the $5 \mathrm{~Hz}-10 \mathrm{~ms}, 5 \mathrm{~Hz}-100 \mathrm{~ms}$, and $50 \mathrm{~Hz}-10 \mathrm{~ms}$ groups showed a significant loss of tyrosine hydroxylase (TH)-positive cells compared to the control group $(* * * p<0.001)$. The $5 \mathrm{~Hz}-10$ $\mathrm{ms}, 5 \mathrm{~Hz}-100 \mathrm{~ms}$, and $50 \mathrm{~Hz}-10 \mathrm{~ms}$ groups were significantly different from the complete model group (** $p<0.01$ ), and the $5 \mathrm{~Hz}$ $10 \mathrm{~ms}$ and $50 \mathrm{~Hz}-10 \mathrm{~ms}$ groups were significantly different from the partial model group $(* p<0.05)$. One-way ANOVA with the Tukey multiple comparison tests.

\section{Statistical Analysis}

All data are presented as mean \pm standard error of mean. All statistical analyses were performed using Prism software (GraphPad, La Jolla, CA, USA). Two-way repeated-measures ANOVA with the Bonferroni post hoc test were conducted to analyze differences in the time-dependent patterns of the stepping tests. Nigral cell counts were analyzed using one-way ANOVA with the Tukey post hoc test.

\section{Results}

\section{Effect of Optical Inhibition of SN on Motor Activity}

All animals in the $5 \mathrm{~Hz}-10 \mathrm{~ms}, 5 \mathrm{~Hz}-100 \mathrm{~ms}$, and 50 $\mathrm{Hz}-10 \mathrm{~ms}$ groups that received hSynapsin $1-N p H R-Y F P$ AAV injections had contralateral forelimb akinesia induced by $16 \mathrm{~h}$ of illumination with 590-nm light. They showed significant decreases in contralateral adjusting steps compared to ipsilateral adjusting steps $(p<0.001$; Fig. $2 d-f)$. On the other hand, the animals in the control group that received hSynapsin 1-YFP AAV injections did not show contralateral forelimb akinesia after $16 \mathrm{~h}$ of illumination with 590-nm light (Fig. 2c). These effects persisted 1 day after the 590-nm light was switched off (Fig. 2c-f). The $5 \mathrm{~Hz}-10 \mathrm{~ms}, 5 \mathrm{~Hz}-100 \mathrm{~ms}$, and $50 \mathrm{~Hz}-10$ 


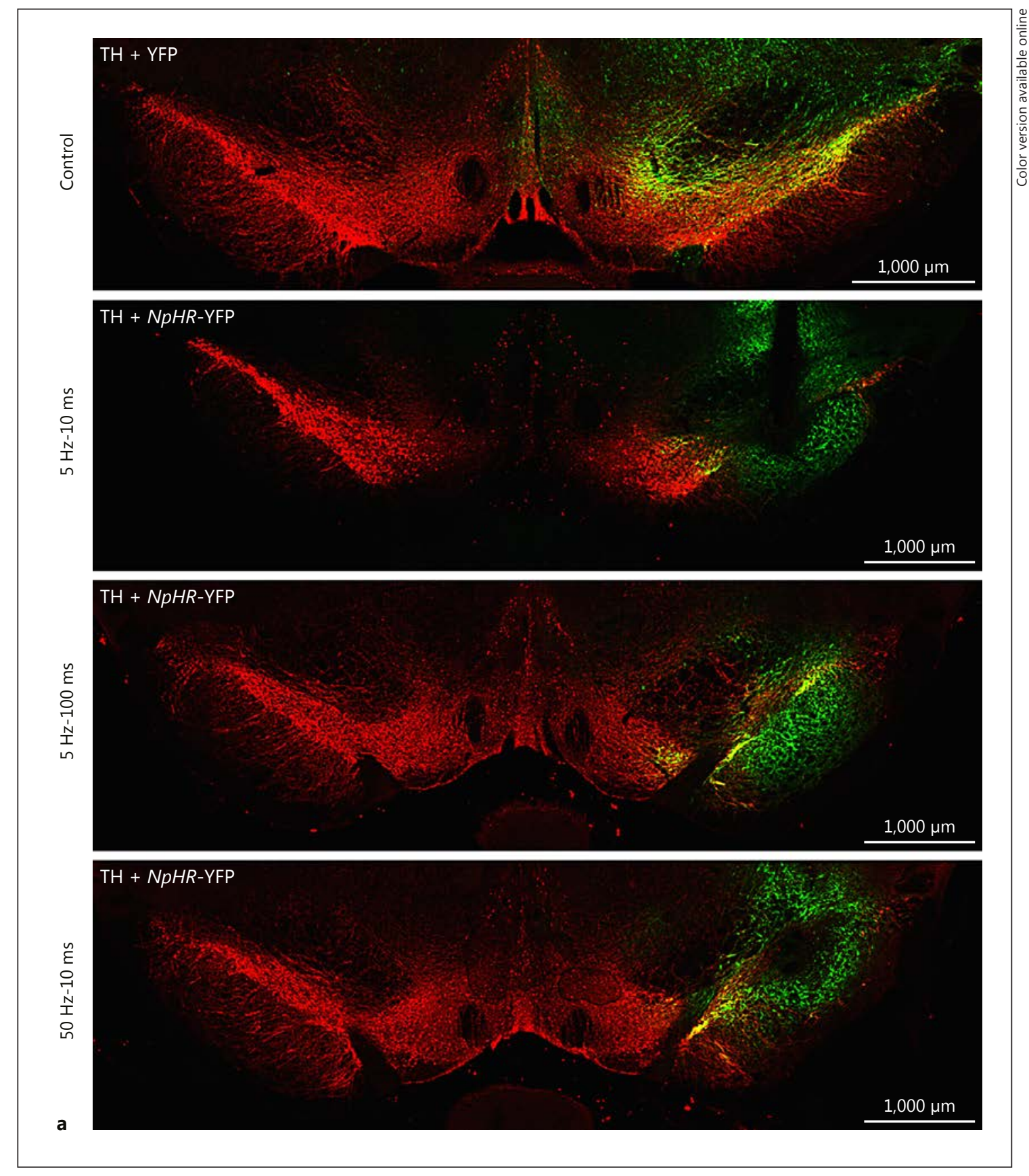

Fig. 4. Immunostaining of the substantia nigra (SN). a, b NpHR-YFP and YFP-expressing cells in all groups were largely detected within the SN area. b The control group showed no change in tyrosine hydroxylase (TH) expression induced by illumination with 590-nm light. However, TH expression was downregulated in the ipsilateral $\mathrm{SN}$ of the $5 \mathrm{~Hz}-10 \mathrm{~ms}, 5 \mathrm{~Hz}-100 \mathrm{~ms}$, and $50 \mathrm{~Hz}-10 \mathrm{~ms}$ groups.

(Figure continued on next page.)

ms groups that received hSynapsin1-NpHR-YFP AAV injections showed significant decreases in the ratio of stepping by the lesioned forelimb compared to the control groups that received hSynapsin1-YFP AAV injec- tions under the light-on condition after $16 \mathrm{~h}$ of $590-\mathrm{nm}$ illumination or $24 \mathrm{~h}$ after the light-off condition $(p<$ 0.001 ; Fig. 2a). When compared with $5 \mathrm{~Hz}-10 \mathrm{~ms}$ of illumination, a higher frequency $(50 \mathrm{~Hz}-10 \mathrm{~ms})$ or greater 


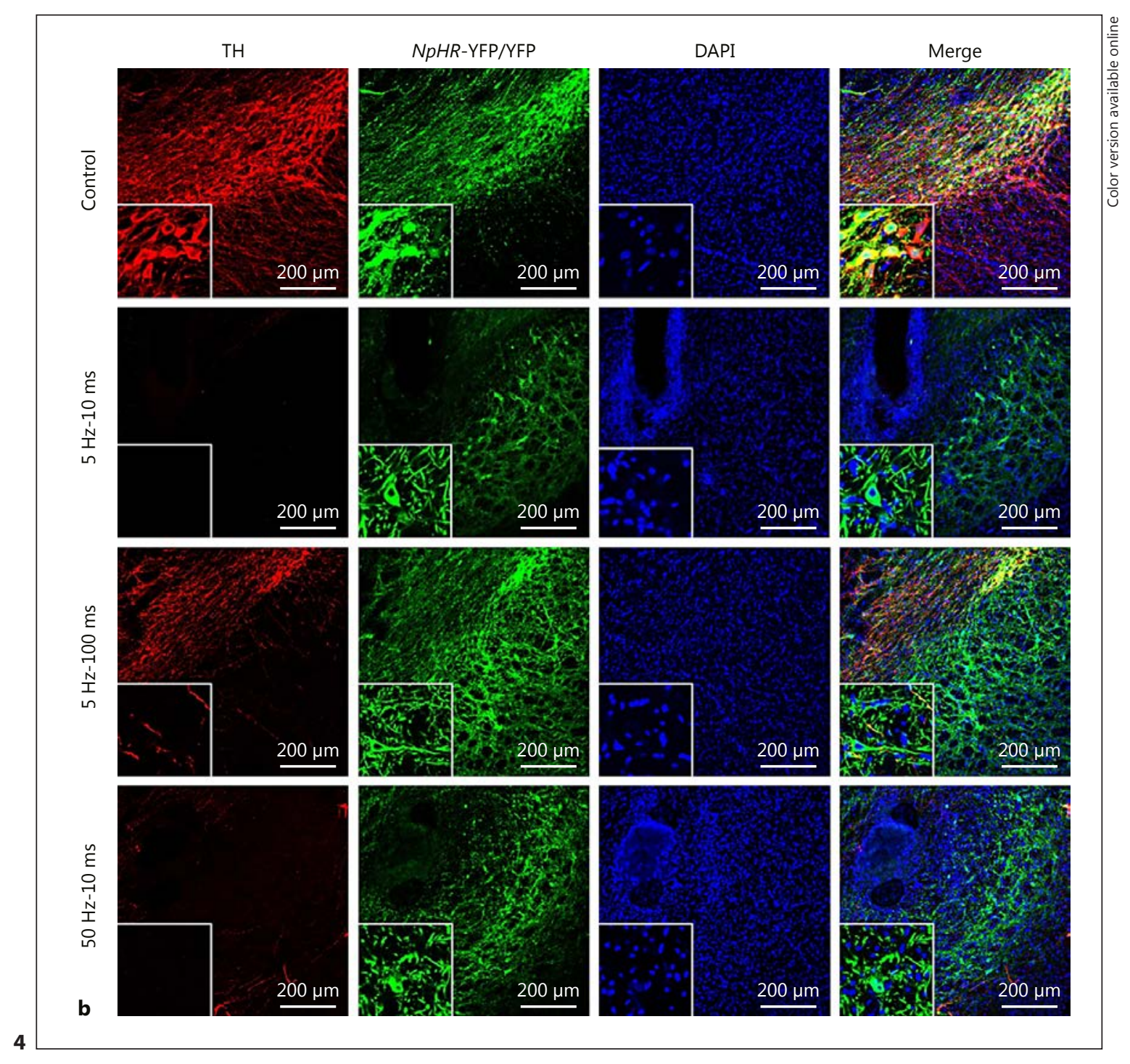

width (5 Hz-100 ms) induced more severe akinesia under the light-on $(p<0.05)$ or light-off ( $p<0.01$; Fig. 2a) condition. Under the light-off condition, the $5 \mathrm{~Hz}-10 \mathrm{~ms}$ group did not show a significant difference compared to the 6-OHDA partial model group, but a significant difference with the 6-OHDA complete model group $(p<0.05$; Fig. 2b). By contrast, the $5 \mathrm{~Hz}-100 \mathrm{~ms}$ and $50 \mathrm{~Hz}-10 \mathrm{~ms}$ groups showed no significant difference when compared with the 6-OHDA partial model group and the complete model group (Fig. 2b).

\section{Effect of Optical Inhibition of SN on TH-Positive Cells}

The ipsilateral SN in the $5 \mathrm{~Hz}-10 \mathrm{~ms}, 5 \mathrm{~Hz}-100 \mathrm{~ms}$, and $50 \mathrm{~Hz}-10 \mathrm{~ms}$ groups showed a significantly considerable loss of TH-positive cells compared to the control group $(p<0.001$; Fig. 3). The loss of TH-positive cells in the SN induced by optical inhibition was less than in the complete group and more than in the partial group. All optical-inhibition groups showed a significant difference compared to the complete model group $(p<0.01)$; the 5 $\mathrm{Hz}-10 \mathrm{~ms}$ and $50 \mathrm{~Hz}-10 \mathrm{~ms}$ groups showed a significant difference compared to the partial model group $(p<0.05)$. However, there was no significant difference among the optical-inhibition groups, indicating a discrepancy with the stepping-test results which demonstrated significant differences among optical-inhibition groups. Immunostaining revealed that $N p H R$-YFP and YFP-expressing cells in all optical-inhibition groups were largely within the SN (Fig. 4a, b). In all optical-inhibition groups, the neuronal action potential was inhibited by illumination 
with 590-nm light within the target area, resulting in the downregulation of TH expression. By contrast, the control group showed no change in TH expression induced by $590-n m$ light illumination.

\section{Discussion}

The pathognomonic features of $\mathrm{PD}$ are the progressive degeneration of DAergic neurons in the $\mathrm{SN}$, the loss of striatal DAergic fibers, and a considerable reduction in striatal dopamine levels. Since 1957, when the neurotoxic agent reserpine was first used to develop an animal model of PD, specific DAergic toxins, such as 6-OHDA (in rats) and 1-methyl-4-phenyl-1,2,3,6-tetrahydropyridine (in primates), that selectively disrupt catecholaminergic neurons, were introduced to develop PD animal models $[7,18]$. Specifically, rat PD models were made by the administration of 6-OHDA into either the SN or MFB for a complete model $[4,7]$, resembling advanced-stage $\mathrm{PD}$, and into the striatum for a partial model that resembles early-to-middle-stage PD [8-11]. When 6-OHDA is injected into the SN or MFB in which DA axons and cell bodies are densely packed, the near-complete degeneration of DAergic neurons in the SN rapidly occurs, beginning within $12 \mathrm{~h}$ and ending within a week of injection [4]. On the other hand, the injection of 6-OHDA into the striatum, which is a much larger structure than the $\mathrm{SN}$ in rats, causes the partial destruction of DAergic axons and terminals within the injected area, followed by the retrograde degeneration of the nigrostriatal system in a much slower and delayed fashion, usually over $4-8$ weeks [7-9, 11,33 . This neurotoxin-based lesioning in the nigra or DAergic pathways is a well-established method for inducing symptoms mimicking those of $\mathrm{PD}$, and represents the characteristic irreversibility of PD. However, PD models using neurotoxins such as 6-OHDA can reproduce only certain fixed stages of the disease, usually either an advanced or early stage, with the drawback that they cannot display the progressive nature of the disease, characteristic of neurodegenerative PD, within 1 individual.

More recently, animal models manipulated to introduce familial PD-related mutant genes ( $\alpha$-synuclein, DJ-1, PINK1, and Parkin) or selectively impair SNc neurons by the conditional deletion of genes that play a key role in the maintenance of the nigrostriatal system (Nurrl and MitoPark) have been developed [14, 34]. These genetic models have proven useful in the study of the role of specific genes and related proteins in the pathophysiology of $\mathrm{PD}$, and they provide a foundation to evaluate potential genetic and pharmacological therapies. However, they have some intrinsic shortcomings. First, a vast majority of PD sporadically occurs by the interplay of multifactorial causes, such as the environment, genetics, and epigenetics, with only $10-20 \%$ attributable to heredity [18, 19]. Therefore, genetic models do not reflect general PD. Second, there are currently $>20$ known causal genes of PD $[13,16,34]$; it is difficult to create a genetic model encompassing all these genes, and a model that has 1 mutant gene or a combination of even several mutant genes is also unlikely to reflect general PD.

Optogenetics, developed in the last decade, is a biological technique that introduces a light-driven ion transport gene via viral vectors into cells, typically neurons, to enable the temporary precise control of the excitatory or inhibitory activity of neurons by inducing the depolarization or hyperpolarization of membrane potential under specific wavelengths of light $[20,21,27]$. As an example of a light-gated ion pump, $N p H R$, which carries chloride ions into neurons, generates sufficient current to enable neural silencing when activated with yellow light, unlike channelrhodopsin, which passively transports cations into neurons under blue light, and thus triggers action potentials [21, 35-37]. For these reasons, the optogenetic technique has been used for functional studies of various neurological diseases [20,21]. Our laboratory introduced $\mathrm{NpHR}$ to inactivate $\mathrm{SNc}$ neurons in this study. Illuminations of 590-nm wavelength from optical fibers inserted into the SNc suppressed DAergic neuronal action potentials, resulting in contralateral forelimb akinesia, resembling the conventional 6-OHDA-induced PD model. The motor symptoms of PD in the optogenetic model appeared within $16 \mathrm{~h}$ of illumination regardless of the illumination condition whereas the partial and complete PD models induced with 6-OHDA took 4 weeks and 1 week, respectively, to express symptoms.

Moreover, we demonstrated that the severity of forelimb akinesia demonstrated by the stepping test in our optogenetic model could be regulated by adjusting the frequency and width of illumination, i.e., the severity of akinesia correlated with the light parameters, frequency and width. When a total value (frequency $\times$ pulse width) of illumination was 10 times higher $(5 \mathrm{~Hz}-10 \mathrm{~ms}$ vs. $5 \mathrm{~Hz}-100$ $\mathrm{ms}$ or $50 \mathrm{~Hz}-10 \mathrm{~ms})$, stepping was worsened by approximately $20 \%$ (a stepping reduction rate of 70 vs. $90 \%$ ). Among the optogenetic animal groups, those illuminated with the low light parameter value $(5 \mathrm{~Hz}-10 \mathrm{~ms})$ showed gait symptoms comparable to the conventional partial PD model in which 6-OHDA is injected into the striatum, but the gait symptoms of the groups illuminated with a high 
light parameter value $(5 \mathrm{~Hz}-100 \mathrm{~ms}$ or $50 \mathrm{~Hz}-10 \mathrm{~ms})$ were similar to those of conventional complete PD models in which 6-OHDA is injected into the MFB. These results suggest that the PD model using optogenetics has the advantages of representing various $P D$ stages in an individual animal by simply adjusting the illumination parameter and more quickly achieving disease manifestation. Our findings also reveal that this animal model could be used to study the mechanisms of PD progression.

When DAergic neurons in the SNc are depolarized, calcium ions move into cells via voltage-sensitive calcium channels, and the increase in intracellular calcium concentrations stimulates $\mathrm{TH}$, the most important rate-limiting enzyme involved in the catecholamine synthesis that determines dopamine levels by catalyzing the hydroxylation of L-tyrosine to L-DOPA (L-3,4,-dihydroxyphenylalanine) $[38,39]$. After L-DOPA is subsequently decarboxylated to dopamine by the aromatic amino acid decarboxylase, dopamine is delivered to the synaptic vesicle with the help of vesicular monoamine transporter 2 (VMAT-2). Dopamine is then released into the synaptic cleft through exocytosis when calcium ions enter the neuron, leading to the transmission of nerve signals [38]. TH expression in DAergic neurons, in particular, is modulated by various conditions and has been reported to be dependent on $\mathrm{SNc}$ neuronal activity $[11,13,33,40-43]$. Thus, the excitation of DAergic neurons induces an increase in TH expression, and the degree of neuronal silencing is equivalent to the decrease in the expression levels of TH [42, 44-48]. Moreover, it has been reported that DAergic neuronal activity controls TH expression and activity through calcium iondependent transcriptional mechanisms and various posttranscriptional mechanisms [38, 42, 49, 50].

In this study, immunofluorescence staining assays showed decreases in TH-positive DAergic neurons in the $\mathrm{SNc}$ of all NpHR-expressing PD models, and this occurred within just $16 \mathrm{~h}$ of illumination with $590-\mathrm{nm}$ light during which $N p H R$ hyperpolarized and silenced DAergic neurons. Though the quantitative changes in $\mathrm{TH}$ activity and DA exocytosis after optogenetic inhibition were not investigated, we assume that neuronal silencing induced by $N p H R$ stimulation would also reduce TH activity and DA exocytosis since, theoretically, TH is activated by the excitation of DAergic neurons and DA exocytosis is triggered by the influx of calcium ion that occurs after an action potential reaches the presynaptic nerve terminal. We consider that the DA level was reduced by a series of these mechanisms, and that our optogenetic model could represent PD manifestation [51]. Meanwhile, PD features due to DAergic neuron inhibition per-

Modulating the Illumination Parameter in an Animal Model to Represent PD Stages sisted not only upon illumination but also $24 \mathrm{~h}$ after the light stimulation was discontinued. We presume that the reason for akinesia lasting $24 \mathrm{~h}$ despite this discontinuation is because TH expression needs a certain amount of time for transcriptional processes to occur. Aumann et al. [42] reported that changes in TH expression began 12$24 \mathrm{~h}$ following the onset of sustained altered stimuli in adult mouse SNc neurons. In contrast, the $\mathrm{TH}$ activity and dopamine exocytosis that were previously suppressed by light-activated $N p H R$ would probably have recovered as soon as the illumination was discontinued because the neuron would have been reactivated immediately if the illumination was interrupted [42]. In other words, we suggest that PD symptoms persisted $24 \mathrm{~h}$ after the discontinuation of illumination because of the sustained reduction in the effects of dopamine due to the delayed $\mathrm{TH}$ expression. We did not investigate when $\mathrm{TH}$ expression recovers after the discontinuation of illumination because we focused here on generating PD symptoms; in future studies, we plan to see how long it takes for the expression of TH to be reestablished. Meanwhile, the degree of inhibition of $\mathrm{TH}$ expression in $\mathrm{SNc}$ neurons did not differ in the low and high illumination value groups and was also not proportional to the severity of PD motor symptoms. The discrepancy between the degree of $\mathrm{TH}$ expression and the stepping-test results suggests that other mechanisms, such as changes in the activities of $\mathrm{TH}$, vesicle VMAT-2, or dopamine transporter by optogenetic inhibition, may contribute further to the dopamine reduction in our optogenetic PD model, and further studies are needed to explain these possible mechanisms.

We have used optogenetic technology to develop a PD model in which the disease stage can be controlled by adjusting the illumination parameters, and it is expected that we will be able to observe changes as the disease progresses within an individual. Future studies are necessary to determine whether the illumination parameters can be varied to develop more diverse disease stages, namely presymptomatic, immediate onset, subchronic, and progressive chronic PD. Moreover, electrophysiologic studies should confirm whether our optogenetic model reproduces the intricate neurochemical deficits of PD. Meanwhile, the reversible nature of the optogenetic inhibition is in conflict with the characteristics of PD which has irreversible neuronal degeneration. Nevertheless, the optogenetic PD model will be able to contribute to the establishment of PD pathogenesis by helping to elucidate the detailed changes in the neuronal firing in the motor circuit at each stage of the disease because we can regulate the disease stage by adjusting the illumination parame- 
ters. Furthermore, this optogenetic PD model is able to reproduce the initial and intermediate stages of the $\mathrm{PD}$ during which DAergic neuronal cell death has not occurred fully but has been functionally repressed. It can therefore be used for research to establish preventive and therapeutic strategies for each stage of the disease. Considering the remarkable technological advancements in optogenetics, we aim to develop more physiologically ideal animal models in the near future to conduct indepth studies of neurological diseases.

\section{Conclusion}

A novel PD animal model using the optogenetic technology developed in this study has the advantage of more quickly representing the various stages of PD by control- ling the illumination parameters. It can be used for the study of the mechanism of progression of PD as well as the management of various disease stages.

\section{Disclosure Statement}

The authors have no financial disclosures and attest that they meet the current ICMJE criteria for authorship.

\section{Funding Sources}

This research was supported by a grant from the Korea Health Technology R\&D Project through the Korea Health Industry Development Institute funded by the Ministry of Health \& Welfare, Republic of Korea (grant No. HI16C2188), and an Asan Life Science Institute Grant (grant No. 16-241) from the Asan Medical Center, Seoul, Republic of Korea.

\section{References}

1 Filion M, Tremblay L, Bedard PJ: Abnormal influences of passive limb movement on the activity of globus pallidus neurons in parkinsonian monkeys. Brain Res 1988;444:165176.

2 Wichmann T, Bergman H, DeLong MR: The primate subthalamic nucleus. III. Changes in motor behavior and neuronal activity in the internal pallidum induced by subthalamic inactivation in the MPTP model of parkinsonism. J Neurophysiol 1994;72:521-530.

-3 Wichmann T, Bergman H, Starr PA, Subramanian T, Watts RL, DeLong MR: Comparison of MPTP-induced changes in spontaneous neuronal discharge in the internal pallidal segment and in the substantia nigra pars reticulata in primates. Exp Brain Res 1999;125: 397-409.

-4 Faull RL, Laverty R: Changes in dopamine levels in the corpus striatum following lesions in the substantia nigra. Exp Neurol 1969;23: 332-340.

5 Perese DA, Ulman J, Viola J, Ewing SE, Bankiewicz KS: A 6-hydroxydopamine-induced selective parkinsonian rat model. Brain Res 1989;494:285-293.

6 Sachs C, Jonsson G: Mechanisms of action of 6-hydroxydopamine. Biochem Pharmacol 1975;24:1-8.

7 Schober A: Classic toxin-induced animal models of Parkinson's disease: 6-OHDA and MPTP. Cell Tissue Res 2004;318:215-224.

8 Przedborski S, Levivier M, Jiang H, Ferreira M, Jackson-Lewis V, Donaldson D, Togasaki DM: Dose-dependent lesions of the dopaminergic nigrostriatal pathway induced by intrastriatal injection of 6-hydroxydopamine. Neuroscience 1995;67:631-647.
9 Berger K, Przedborski S, Cadet JL: Retrograde degeneration of nigrostriatal neurons induced by intrastriatal 6-hydroxydopamine injection in rats. Brain Res Bull 1991;26:301-307.

10 Ichitani Y, Okamura H, Matsumoto Y, Nagatsu I, Ibata Y: Degeneration of the nigral dopamine neurons after 6-hydroxydopamine injection into the rat striatum. Brain Res 1991; 549:350-353.

11 Bjorklund A, Rosenblad C, Winkler C, Kirik D: Studies on neuroprotective and regenerative effects of GDNF in a partial lesion model of Parkinson's disease. Neurobiol Dis 1997;4: 186-200.

12 Lee CS, Sauer H, Bjorklund A: Dopaminergic neuronal degeneration and motor impairments following axon terminal lesion by instrastriatal 6-hydroxydopamine in the rat. Neuroscience 1996;72:641-653.

13 Antony PM, Diederich NJ, Balling R: Parkinson's disease mouse models in translational research. Mamm Genome 2011;22:401-419.

14 Dawson TM, Ko HS, Dawson VL: Genetic animal models of Parkinson's disease. Neuron 2010;66:646-661.

15 Fujioka S, Wszolek ZK: Update on genetics of parkinsonism. Neurodegener Dis 2012;10: 257-260.

16 Klein C, Westenberger A: Genetics of Parkinson's disease. Cold Spring Harb Perspect Med 2012;2:a008888.

17 Verstraeten A, Theuns J, Van Broeckhoven C: Progress in unraveling the genetic etiology of Parkinson disease in a genomic era. Trends Genet 2015;31:140-149.

18 Dauer W, Przedborski S: Parkinson's disease: mechanisms and models. Neuron 2003;39: 889-909.
19 Gasser T, Hardy J, Mizuno Y: Milestones in PD genetics. Mov Disord 2011;26:1042-1048. 20 Boyden ES: A history of optogenetics: the development of tools for controlling brain circuits with light. F1000 Biol Rep 2011;3:11.

21 Boyden ES, Zhang F, Bamberg E, Nagel G, Deisseroth K: Millisecond-timescale, genetically targeted optical control of neural activity. Nat Neurosci 2005;8:1263-1268.

22 Schmidt D, Cho YK: Natural photoreceptors and their application to synthetic biology. Trends Biotechnol 2015;33:80-91.

$\longrightarrow 23$ Tischer D, Weiner OD: Illuminating cell signalling with optogenetic tools. Nat Rev Mol Cell Bio 2014;15:551-558.

24 Zhang K, Cu BX: Optogenetic control of intracellular signaling pathways. Trends Biotechnol 2015;33:92-100.

25 Grusch M, Schelch K, Riedler R, Reichhart E, Differ C, Berger W, Ingles-Prieto A, Janovjak $\mathrm{H}$ : Spatio-temporally precise activation of engineered receptor tyrosine kinases by light. EMBO J 2014;33:1713-1726.

26 Idevall-Hagren O, Dickson EJ, Hille B, Toomre DK, De Camilli P: Optogenetic control of phosphoinositide metabolism. Proc Natl Acad Sci USA 2012;109:E2316-E2323.

27 Park S, Koppes RA, Froriep UP, Jia X, Achyuta AK, McLaughlin BL, Anikeeva P: Optogenetic control of nerve growth. Sci Rep 2015;5: 9669.

28 Yoon HH, Min J, Hwang E, Lee CJ, Suh JK, Hwang O, Jeon SR: Optogenetic inhibition of the subthalamic nucleus reduces levodopainduced dyskinesias in a rat model of Parkinson's disease. Stereotact Funct Neurosurg 2016;94:41-53. 
-29 Yoon HH, Park JH, Kim YH, Min J, Hwang E, Lee CJ, Suh JK, Hwang O, Jeon SR: Optogenetic inactivation of the subthalamic nucleus improves forelimb akinesia in a rat model of Parkinson disease. Neurosurgery 2014;74: 533-540; discussion 540-531.

- 30 Kollensperger M, Stefanova N, Pallua A, Puschban Z, Dechant G, Hainzer M, Reindl M, Poewe W, Nikkhah G, Wenning GK: Striatal transplantation in a rodent model of multiple system atrophy: effects on L-dopa response. J Neurosci Res 2009;87:1679-1685.

- 31 Decressac M, Mattsson B, Bjorklund A: Comparison of the behavioural and histological characteristics of the 6-OHDA and alphasynuclein rat models of Parkinson's disease. Exp Neurol 2012;235:306-315.

32 Paille V, Henry V, Lescaudron L, Brachet P, Damier P: Rat model of Parkinson's disease with bilateral motor abnormalities, reversible with levodopa, and dyskinesias. Mov Disord 2007;22:533-539.

33 Sauer H, Oertel WH: Progressive degeneration of nigrostriatal dopamine neurons following intrastriatal terminal lesions with 6-hydroxydopamine: a combined retrograde tracing and immunocytochemical study in the rat. Neuroscience 1994;59:401-415.

- 34 Gubellini P, Kachidian P: Animal models of Parkinson's disease: an updated overview. Rev Neurolog 2015;171:750-761.

- 35 Chuong AS, Miri ML, Busskamp V, Matthews GA, Acker LC, Sorensen AT, Young A, Klapoetke NC, Henninger MA, Kodandaramaiah SB, Ogawa M, Ramanlal SB, Bandler RC, Allen BD, Forest CR, Chow BY, Han X, Lin Y, Tye KM, Roska B, Cardin JA, Boyden ES: Noninvasive optical inhibition with a redshifted microbial rhodopsin. Nat Neurosci 2014;17:1123-1129.
36 Han X, Boyden ES: Multiple-color optical activation, silencing, and desynchronization of neural activity, with single-spike temporal resolution. PLoS One 2007;2:e299.

37 Zhang F, Wang LP, Brauner M, Liewald JF, Kay K, Watzke N, Wood PG, Bamberg E, Nagel G, Gottschalk A, Deisseroth K: Multimodal fast optical interrogation of neural circuitry. Nature 2007;446:633-639.

38 Daubner SC, Le T, Wang S: Tyrosine hydroxylase and regulation of dopamine synthesis. Arch Biochem Biophys 2011;508:1-12.

39 Haavik J, Martinez A, Flatmark T: pH-dependent release of catecholamines from tyrosine hydroxylase and the effect of phosphorylation of Ser-40. FEBS Lett 1990;262:363-365.

40 Aumann TD, Gantois I, Egan K, Vais A, Tomas D, Drago J, Horne MK: SK channel function regulates the dopamine phenotype of neurons in the substantia nigra pars compacta. Exp Neurol 2008;213:419-430.

41 Bowenkamp KE, David D, Lapchak PL, Henry MA, Granholm AC, Hoffer BJ, Mahalik TJ: 6-hydroxydopamine induces the loss of the dopaminergic phenotype in substantia nigra neurons of the rat. A possible mechanism for restoration of the nigrostriatal circuit mediated by glial cell line-derived neurotrophic factor. Exp Brain Res 1996;111:1-7.

42 Aumann TD, Egan K, Lim J, Boon WC, Bye CR, Chua HK, Baban N, Parish CL, Bobrovskaya L, Dickson P, Horne MK: Neuronal activity regulates expression of tyrosine hydroxylase in adult mouse substantia nigra pars compacta neurons. J Neurochem 2011;116: 646-658.

43 Gash DM, Zhang Z, Ovadia A, Cass WA, Yi A, Simmerman L, Russell D, Martin D, Lapchak PA, Collins F, Hoffer BJ, Gerhardt GA: Functional recovery in parkinsonian monkeys treated with GDNF. Nature 1996;380: 252-255.
44 Dreyfus CF, Friedman WJ, Markey KA, Black IB: Depolarizing stimuli increase tyrosine hydroxylase in the mouse locus coeruleus in culture. Brain Res 1986;379:216-222.

45 Menezes A, Zeman R, Sabban E: Involvement of intracellular or extracellular calcium in activation of tyrosine hydroxylase gene expression in PC12 cells. J Neurochem 1996;67: 2316-2324.

46 Stachowiak MK, Goc A, Hong JS, Poisner A, Jiang HK, Stachowiak EK: Regulation of tyrosine hydroxylase gene expression in depolarized non-transformed bovine adrenal medullary cells: second messenger systems and promoter mechanisms. Brain Res Mol Brain Res 1994;22:309-319.

47 Stone DM, Grillo M, Margolis FL, Joh TH, Baker H: Differential effect of functional olfactory bulb deafferentation on tyrosine hydroxylase and glutamic acid decarboxylase messenger RNA levels in rodent juxtaglomerular neurons. J Comp Neurol 1991;311:223233.

48 Zigmond RE: The long-term regulation of ganglionic tyrosine hydroxylase by preganglionic nerve activity. Fed Proc 1980;39:30033008.

49 Flavell SW, Greenberg ME: Signaling mechanisms linking neuronal activity to gene expression and plasticity of the nervous system. Annu Rev Neurosci 2008;31:563-590.

50 Doskeland AP, Flatmark T: Ubiquitination of soluble and membrane-bound tyrosine hydroxylase and degradation of the soluble form. Eur J Biochem 2002;269:1561-1569.

-51 Parker KL, Kim Y, Alberico SL, Emmons EB, Narayanan NS: Optogenetic approaches to evaluate striatal function in animal models of Parkinson disease. Dialogues Clin Neurosci 2016;18:99-107. 